\title{
An asymptotic preserving scheme for the Kac model of the Boltzmann equation in the diffusion limit
}

\author{
Mounir Bennoune ${ }^{1}$, Mohammed Lemou ${ }^{2}$, Luc Mieussens ${ }^{3}$
}

\begin{abstract}
In this paper we propose a numerical scheme to solve the Kac model of the Boltzmann equation for multiscale rarefied gas dynamics. This scheme is uniformly stable with respect to the Knudsen number, consistent with the fluid-diffusion limit for small Knudsen numbers, and with the Kac equation in the kinetic regime. Our approach is based on the micro-macro decomposition which leads to an equivalent formulation of the Kac model that couples a kinetic equation with macroscopic ones. This method is validated with various test cases and compared to other standard methods.

Key words. Kac equation, diffusion limit, Hilbert expansion, asymptotic preserving schemes, micro-macro decomposition.
\end{abstract}

AMS subject classifications. 65M06, 35B25, 82C80, 41A60

\section{Introduction}

In this work, we extend a method developped in [3] in order to construct asymptotic preserving schemes (AP) for kinetic equations. The AP property means that the scheme is uniformly stable and accurate with respect to the Knudsen number. In particular, it is consistent with the fluid limit when the Knudsen number is small (see [10], for instance).

There are different approaches to construct such schemes: generally, these methods are based on a splitting method which consists in solving the collision and the transport part separately. We refer for instance to the following recent papers $[11,9,5,22,23,13,15,17$, $12,10,21,16]$.

Our strategy is different, since it consists in rewritting the kinetic equation as a coupled system of kinetic part and macroscopic one, by using the micro-macro decomposition of the distribution function. Indeed, this function is decomposed into its corresponding (Maxwellian) equilibrium distribution plus the deviation. By using a classical projection technique (like in the Chapmann-Enskog expansion), we obtain an evolution equation for

\footnotetext{
${ }^{1}$ Institut de Mathématiques de Toulouse (UMR 5219), Université Paul Sabatier, 118, Route de Narbonne, 31062 Toulouse cedex 9, France (bennoune@mip.ups-tlse.fr)

${ }^{2}$ Institut de Recherche Mathématique de Rennes (IRMAR) (UMR6625), Université Rennes I, Campus de Beaulieu 35042 Rennes cedex (France), (mohammed.lemou@univ-rennes1.fr)

${ }^{3}$ Institut de Mathématiques de Bordeaux (UMR 5251), Université de Bordeaux, 351, cours de la Libération,33405 Talence cedex, France (Luc.Mieussens@math.u-bordeaux1.fr)
} 
the macroscopic parameters of the Maxwellian coupled to a kinetic equation for the nonequilibrium part. This idea has been used to design a numerical scheme that preserves both the compressible Euler and Navier-Stokes asymptotics for the Boltzmann equation of rarefied gas dynamics in [3], and also to obtain asymptotic preserving schemes in the diffusion limit for linear kinetic equations [19]. Although our approach is rather general to apply to a very large class of collision operators, the numerical tests shown in our previous work were obtained with very simple models (the BGK model for [3] and the one group neutron transport equation for [19]).

In this paper, our aim is to prove that our approach can be applied to more complex collision operators. Of course, the ultimate model would be the full Boltzmann equation. However, numerically solving the Boltzmann equation is known to be rather difficult (it is computationnaly expensive, and implementing any discretiation of the collision operator takes a lot of time). As a first step into this direction, we apply our approach to the Kac equation. This model is much simpler that the Boltzmann equation (it is one dimensional), but it has the same quadratic structure, while the models used in our previous works were only relaxation operators. The main consequence of this structure is that the linearized collision operator which is induced by the micro-macro decomposition is not diagonal. However, contrary to the Boltzmann equation, the natural fluid limit of the Kac model is a non linear diffusion equation.

The outline of this paper is the following. In section 2, we present the Kac model and derive its diffusion limit by using the classical Hilbert expansion. The micro-macro decomposition and the corresponding formulation of the Kac equation are presented in section 3. Our numerical scheme is presented in section 4. Finally, before a conclusion, the properties of our method are demonstrated with several numerical tests in section 5 .

\section{The Kac model and its properties}

\subsection{The non-homogeneous Kac equation}

The non-homogeneous Kac equation (introduced in [14], see also [20]) is:

$$
\partial_{t} f+v \partial_{x} f=Q(f, f)
$$

where $f=f(t, x, v)$ is the distribution function which depends on time $t \geq 0$, on the position of particles $x \in \mathbb{R}$ and on their velocity $v \in \mathbb{R}$.

The right-hand side models the collision between particles by the collisional operator $Q$, which is a bilinear functional acting only on the velocity dependence of the distribution function $f$. More precisely, it writes

$$
Q(f, f)(v)=\frac{1}{2 \pi} \int_{0}^{2 \pi} \int_{\mathbb{R}}\left[f\left(v^{\prime}\right) f\left(v_{*}^{\prime}\right)-f(v) f\left(v_{*}\right)\right] d \theta d v_{*},
$$


where $\left(v^{\prime}, v_{*}^{\prime}\right)$ are the post-collisional velocities obtained from the pre-collisional velocities $\left(v, v_{*}\right)$ by a random rotation $\theta \in\left[0,2 \pi\left[\right.\right.$ in $\mathbb{R}^{2}$, such that

$$
\left(v^{\prime}, v_{*}^{\prime}\right)=\left(v \cos \theta-v_{*} \sin \theta, v \sin \theta+v_{*} \cos \theta\right)
$$

It is clear that pre- and post-collisional velocities satisfy the following relation $v^{2}+v_{*}^{2}=$ $v^{\prime 2}+v_{*}^{\prime 2}$, which is nothing but the conservation of the kinetic energy of the pair of particles during a collision.

The collision operator has important physical properties that are detailed below. For clarity, we use the following notations in all what follows:

$$
m(v)=\left(1, \frac{v^{2}}{2}\right)^{T}, \quad \text { and } \quad\langle g\rangle=\int_{\mathbb{R}} g(v) d v
$$

for any scalar or vector function $g=g(v)$. Then it is classical to prove that $Q$ satisfies:

1. local conservation of mass and energy.

$$
\langle m Q(f, f)\rangle=0, \quad \forall f \geq 0 .
$$

2. entropy inequality

$$
\langle Q(f, f) \log (f)\rangle \leq 0, \quad \forall f \geq 0 .
$$

3. non-negative equilibrium functions $f$, i.e., such that $Q(f, f)=0$, are the Maxwellian distributions given by

$$
M(v)=\frac{\rho}{(2 \pi T)^{\frac{1}{2}}} \exp \left(-\frac{v^{2}}{2 T}\right),
$$

where $\rho$ and $T$ are the density and the temperature associated to $M$ by the relation

$$
\langle m M\rangle=\left(\rho, \frac{1}{2} \rho T\right) .
$$

We note that the conservation of momentum is not satisfied by the Kac collisional operator. Proofs of these properties and more theoritical results about the Kac equation can be found in $[6,8]$.

\subsection{Diffusion limit}

When the number of collisions becomes large, the mean free path (the distance travelled by a particle between two collisions) becomes small as compared to a characteristic lenght of the computational domain. It is therefore interesting to use a new set of macroscopic variables $x^{\prime}$ and $t^{\prime}$ according to

$$
x^{\prime}=\varepsilon x, \quad t^{\prime}=\varepsilon^{2} t,
$$


where $\varepsilon$ is the Knudsen number, defined as the ratio between the mean free path and the characteristic macroscopic length. This choice is different from the usual scaling to derive Euler or Navier-Stokes equation from the Boltzmann equation (where $t^{\prime}=\varepsilon t$ ). It is mainly guided by the structure of the Kac collision operator: roughly speaking, as it is shown below, the macroscopic flux of particles in the limit of small $\varepsilon$ becomes very small, which means that the macroscopic flow is slow, and that a large macroscopic time scale must be chosen.

Indeed, with these new variables, we get the equation:

$$
\partial_{t} f+\frac{1}{\varepsilon} v \partial_{x} f=\frac{1}{\varepsilon^{2}} Q(f, f)
$$

Thus when $\varepsilon$ goes to 0 , the distribution function $f$ tends towards $f_{0}$ which is a solution to $Q\left(f_{0}, f_{0}\right)=0$. This implies that $f_{0}$ is a local Maxwellian $M_{0}$ with zero mean velocity which is determined by the parameters mass density $\rho_{0}$ and energy $E_{0}=\frac{1}{2} \rho_{0} T_{0}$, accordingly to relation (6). The diffusion limit is then determined by the macroscopic equations satisfied by $\rho_{0}$ and $E_{0}$. In order to obtain these equations, we use the classical Hilbert expansion: $f$ is formally expanded into the following series:

$$
f=M_{0}+\varepsilon f_{1}+\varepsilon^{2} f_{2}+\ldots
$$

Calculating the different terms of this expansion requires the use the linearized operator $\mathcal{L}_{M_{0}}$ of $Q$ arround the Maxwellian $M_{0}$. It is defined by

$$
\begin{aligned}
\mathcal{L}_{M_{0}} g & =2 Q\left(M_{0}, g\right) \\
= & \frac{1}{2 \pi} \int_{\mathbb{R}} \int_{0}^{2 \pi}\left[M_{0}\left(v^{\prime}\right) g\left(v_{*}^{\prime}\right)+g\left(v^{\prime}\right) M_{0}\left(v_{*}^{\prime}\right)-M_{0}(v) g\left(v_{*}\right)-g(v) M_{0}\left(v_{*}\right)\right] d \theta d v_{*}, \forall g,
\end{aligned}
$$

where $\left(v^{\prime}, v_{*}^{\prime}\right)$ are associated to $\left(v, v_{*}\right)$ by relation $(3)$. The derivation of the diffusion limit $\left(\varepsilon \rightarrow 0\right.$ in (8)) depends naturally of the properties of $\mathcal{L}_{M_{0}}$. Let us recall some important properties of this operator. For this, we define the Hilbert space $L_{M_{0}}^{2}=\left\{\varphi=\varphi(v)\right.$ tel que $\varphi M_{0}^{-1} \in$ $\left.L^{2}\left(\mathbb{R}_{v}\right)\right\}$ endowed with the weighted scalar product

$$
(\varphi, \psi)_{M_{0}}:=\left\langle\varphi \psi M_{0}^{-1}\right\rangle
$$

Thus, the properties of $Q$ given above induce the following properties of $\mathcal{L}_{M_{0}}$ :

1. conservation of mass and energy: $\left\langle m \mathcal{L}_{M_{0}} g\right\rangle=0, \forall g$.

2. $\mathcal{L}_{M_{0}}$ is a non-positive self-adjoint operator on $L_{M_{0}}^{2}$.

3. the null space of $\mathcal{L}_{M_{0}}$ is $\mathcal{K} e r\left(\mathcal{L}_{M_{0}}\right)=\operatorname{Vect}\left\{M_{0}, v^{2} M_{0}\right\}$.

4. the orthogonal of its null space is $\mathcal{R}\left(\mathcal{L}_{M_{0}}\right)=\left(\mathcal{K} e r\left(\mathcal{L}_{M_{0}}\right)\right)^{\perp}=\left\{\varphi \in L^{2}\left(\mathbb{R}_{v}\right)\right.$ such that $\langle m \varphi\rangle=$ $0\}$. 
5. for every $g \in \mathcal{R}\left(\mathcal{L}_{M_{0}}\right)$, the equation $\mathcal{L}_{M_{0}} f=g$ has a unique solution in $\mathcal{R}\left(\mathcal{L}_{M_{0}}\right)$, denoted by $f=\mathcal{L}_{M_{0}}^{-1} g$.

Now, inserting expansion (9) in (8), we obtain

$$
\begin{aligned}
\left(\partial_{t}\right. & \left.+\frac{1}{\varepsilon} v \partial_{x}\right)\left(M_{0}+\varepsilon f_{1}+\varepsilon^{2} f_{2}+O\left(\varepsilon^{3}\right)\right) \\
& =\frac{1}{\varepsilon^{2}}\left[Q\left(M_{0}, M_{0}\right)+\varepsilon 2 Q\left(M_{0}, f_{1}\right)+\varepsilon^{2}\left(2 Q\left(M_{0}, f_{2}\right)+Q\left(f_{1}, f_{1}\right)\right)+O\left(\varepsilon^{3}\right)\right] .
\end{aligned}
$$

By identification of coefficients with the same powers of $\varepsilon$, we find the following equations:

$$
\begin{array}{ll}
\varepsilon^{-2}: & Q\left(M_{0}, M_{0}\right)=0, \\
\varepsilon^{-1}: & v \partial_{x} M_{0}=\mathcal{L}_{M_{0}} f_{1}, \\
\varepsilon^{0}: & \partial_{t} M_{0}+v \partial_{x} f_{1}=\mathcal{L}_{M_{0}} f_{2}+Q\left(f_{1}, f_{1}\right) .
\end{array}
$$

Equation (11) is obviously satisfied. Now, noticing that $\left\langle v m \partial_{x} M_{0}\right\rangle=0$, properties 4 and 5 above prove that a solution of (12) is

$$
f_{1}=\mathcal{L}_{M_{0}}^{-1}\left(v \partial_{x} M_{0}\right)
$$

Finally, equation (13) has a solution $f_{2}$ if and only if the following solvability condition is satisfied:

$$
\left\langle m\left[\partial_{t} M_{0}+v \partial_{x} f_{1}-Q\left(f_{1}, f_{1}\right)\right]\right\rangle=0 .
$$

By replacing $f_{1}$ by its expression given by (14) and using the conservation property of mass and energy satisfied by $Q$, the previous equation can be simply written as

$$
\partial_{t}\left(\begin{array}{c}
\rho_{0} \\
E_{0}
\end{array}\right)+\partial_{x}\left\langle v m \mathcal{L}_{M_{0}}^{-1}\left(v \partial_{x} M_{0}\right)\right\rangle=0
$$

This is a system of diffusion equations for $\rho_{0}$ and $E_{0}$ which is the diffusion limit of the Kac equation.

By using classical algebra, the fluxes can be analytically computed to find the following explicit form of the diffusion limit (see details in [2]):

$$
\partial_{t}\left(\begin{array}{c}
\rho_{0} \\
E_{0}
\end{array}\right)-\partial_{x}\left(\begin{array}{c}
T_{0} \partial_{x} \log \left(\rho_{0}\right)+\partial_{x} T_{0} \\
\frac{3}{2}\left[T_{0}^{2} \partial_{x} \log \left(\rho_{0}\right)+\partial_{x} T_{0}^{2}\right]
\end{array}\right)=0
$$

\section{The micro-macro decomposition of the Kac equation}

\subsection{The micro-macro decomposition}

Let $f$ be a solution of the Kac equation (8). We decompose $f$ as follows

$$
f=M(U)+\varepsilon g
$$


with

$$
U=\langle m f\rangle=\left(\rho, E=\frac{1}{2} \rho u^{2}+\frac{1}{2} \rho T\right),
$$

where $\rho, u$ and $E$ are the mass density, the kinetic mean velocity, and the energy of the distribution function $f$. Here, $M(U)$ is the centered Maxwellian with the same mass and energy as $f$, that is to say $\langle m M(U)\rangle=U$. Note that $M(U)$ has not the same mean velocity as $f$ in general, and is not defined as in (6) (we mean that the temperature of $M(U)$ is not $T)$. Such Maxwellian will be denoted $M=M(U)$ when no confusion is possible. Also note that $M$ depends on $\varepsilon$ and is not the limit of $f$ when $\varepsilon \rightarrow 0$. We now insert decomposition (17) into equation (8) to get

$$
\partial_{t} M+\varepsilon \partial_{t} g+\frac{1}{\varepsilon} v \partial_{x} M+v \partial_{x} g=\frac{1}{\varepsilon^{2}} Q(M+\varepsilon g, M+\varepsilon g) .
$$

By using the bilinearity of $Q$ and the fact that $Q(M, M)=0$, we obtain

$$
Q(M+\varepsilon g, M+\varepsilon g)=Q(M, M)+2 \varepsilon Q(M, g)+\varepsilon^{2} Q(g, g)=\varepsilon \mathcal{L}_{M} g+\varepsilon^{2} Q(g, g) .
$$

Therefore, equation (18) becomes

$$
\partial_{t} M+\varepsilon \partial_{t} g+\frac{1}{\varepsilon} v \partial_{x} M+v \partial_{x} g=\frac{1}{\varepsilon} \mathcal{L}_{M} g+Q(g, g) .
$$

In order to obtain two evolution equations, one for the equilibrium part $M$ and the other one for the deviation $g$, we now use a projection technique to separate $M$ and $g$. More precisely, let consider the Hilbert space $L_{M}^{2}=\left\{\varphi\right.$ such that $\left.\varphi M^{-\frac{1}{2}} \in L^{2}(\mathbb{R})\right\}$ endowed with the weighted scalar product

$$
(\varphi, \psi)_{M}=\left\langle\varphi \psi M^{-1}\right\rangle
$$

and let $\Pi_{M}$ be the orthogonal projection operator in $L_{M}^{2}$ onto $\operatorname{Ker}\left(\mathcal{L}_{M}\right)$. Thus, $\Pi_{M}$ is characterised by:

$$
\left\langle m\left(\Pi_{M}(\varphi)-\varphi\right)\right\rangle=0, \forall \varphi \in L_{M}^{2} .
$$

To simplify the computations, we consider the orthogonal basis of $\mathcal{K} e r\left(\mathcal{L}_{M}\right)$

$$
\mathcal{B}=\left\{\frac{1}{\rho} M,\left(\frac{v^{2}}{2 \tilde{T}}-\frac{1}{2}\right) \frac{1}{\rho} M\right\} .
$$

Here, $\tilde{T}$ is the temperature of the Maxwellian $M$. A simple calculation leads to the following expression of the projection of any function $\varphi \in L_{M}^{2}$ :

$$
\Pi_{M}(\varphi)=\frac{1}{\rho}\left[\langle\varphi\rangle+2\left\langle\left(\frac{v^{2}}{2 \tilde{T}}-\frac{1}{2}\right) \varphi\right\rangle\left(\frac{v^{2}}{2 \tilde{T}}-\frac{1}{2}\right)\right] M .
$$

The following proposition gives some elementary properties $\Pi_{M}$ (the proof is easy and is left to the reader): 
Proposition 3.1. Let $M$ and $g$ defined by decomposition (17). Then, we have

$$
\left(I-\Pi_{M}\right)\left(\partial_{t} M\right)=\Pi_{M}(g)=\Pi_{M}\left(\partial_{t} g\right)=\Pi_{M}(Q(g, g))=\Pi_{M}\left(\mathcal{L}_{M} g\right)=0 .
$$

Now, we apply the projection $I-\Pi_{M}$ to equation (19). By using property 3.1, we obtain

$$
\partial_{t} g+\frac{1}{\varepsilon}\left(I-\Pi_{M}\right)\left(v \partial_{x} g\right)-\frac{1}{\varepsilon} Q(g, g)=\frac{1}{\varepsilon^{2}}\left[\mathcal{L}_{M} g-\left(I-\Pi_{M}\right)\left(v \partial_{x} M\right)\right] .
$$

Note that since $\partial_{x} M$ is even w.r.t. $v$, then $\left\langle v m \partial_{x} M\right\rangle=0$. Therefore, $\Pi_{M}\left(v \partial_{x} M\right) \in \mathcal{R}\left(\mathcal{L}_{M}\right) \cap$ $\operatorname{Ker}\left(\mathcal{L}_{M}\right)$ and then $\Pi_{M}\left(v \partial_{x} M\right)=0$. Thus, equation (21) becomes

$$
\partial_{t} g+\frac{1}{\varepsilon}\left(I-\Pi_{M}\right)\left(v \partial_{x} g\right)-\frac{1}{\varepsilon} Q(g, g)=\frac{1}{\varepsilon^{2}}\left[\mathcal{L}_{M} g-v \partial_{x} M\right]
$$

Now, by multiplying equation (19) by the vector of collisional invariants $m$ and then integrating with respect to velocity variable, we find

$$
\partial_{t} U+\partial_{x}\langle v m g\rangle=0
$$

Equations (22) and (23) are the micro-macro formulation of the Kac equation (8) that we want to use to desing our AP scheme. The following proposition shows that this formulation is indeed equivalent to the Kac equation (see the proof in appendix A).

Proposition 3.2. With the same previous notations, $f=M(U)+\varepsilon g$ is a solution of the Kac equation (8) with the initial data $f(t=0)=f_{0}$ if and only if the couple $(U, g)$ defined by $U=\langle m f\rangle$ and $g=\frac{1}{\varepsilon}(f-M(U))$ is a solution of the coupled system (22)-(23) with the associated initial data $U(t=0)=\left\langle m f_{0}\right\rangle$ and $g(t=0)=\frac{1}{\varepsilon}\left(f_{0}-M(U(t=0))\right)$.

Remark 3.1. The procedure described above is more or less the classical Chapman-Enskog expansion. While this expansion can be developped in many very different ways, the advantage of our rather mathematical procedure (close to the one detailed in [1]) is very well adapted to numerics, at is will bee seen in section 4 .

\subsection{Diffusion limit}

Here, we rapidly show that the micro-macro formulation (22)-(23) of the Kac equation allows to obtain very easily the diffusion limit (16) as $\varepsilon$ goes to 0 , without using the Hilbert expansion. Indeed, equation (22) gives

$$
\mathcal{L}_{M} g=v \partial_{x} M(U)+O(\varepsilon),
$$

which implies that

$$
g=\mathcal{L}_{M}^{-1}\left(v \partial_{x} M(U)\right)+O(\varepsilon) .
$$

By replacing this expression in (23), we find

$$
\partial_{t} U+\partial_{x}\left\langle v m \mathcal{L}_{M}^{-1}\left(v \partial_{x} M(U)\right)\right\rangle=O(\varepsilon) .
$$

At the limit $\varepsilon$ tends to 0 , one then get the system of diffusion equations

$$
\partial_{t} U_{0}+\partial_{x}\left\langle v m \mathcal{L}_{M_{0}}^{-1}\left(v \partial_{x} M\left(U_{0}\right)\right)\right\rangle=0,
$$

which leads, by setting $U_{0}=\left(\rho_{0}, \frac{1}{2} \rho_{0} T_{0}\right)$, to system (15), hence to the diffusion equations (16). 


\section{Numerical approximation of the micro-macro formu- lation}

For clarity, we separately detail in this section the time, space, and velocity discretizations.

\subsection{Implicit time discretization}

Here, we start by constructing a time discretization of the coupled system (22)-(23). For this, let $\Delta t$ be a fixed time step and $t_{n}=n \Delta t, n \in \mathbb{N}$ a discrete time. We then denote by $\left(U^{n}\right)_{n}$ and $\left(g^{n}\right)_{n}$ two sequences that approximate $U$ and $g$ respectively, that is: $U^{n}(x) \approx$ $U\left(t_{n}, x\right), \quad g^{n}(x, v) \approx g\left(t_{n}, x, v\right)$. The idea of discretization is the same used in [3] for the Boltzmann-BGK equation. In the kinetic part which is an evolution equation on $g$, the main difficulty is that the term $\varepsilon^{-2} \mathcal{L}_{M} g$ becomes stiff when $\varepsilon \rightarrow 0$. To remove this stiffness, we take an implicit time discretization of this term while all other quantities, even $v \partial_{x} M$ which is considered only as a source term, are kept explicit. We obtain

$$
\frac{g^{n+1}-g^{n}}{\Delta t}+\frac{1}{\varepsilon}\left(I-\Pi_{M^{n}}\right)\left(v \partial_{x} g^{n}\right)-\frac{1}{\varepsilon} Q\left(g^{n}, g^{n}\right)=\frac{1}{\varepsilon^{2}}\left[\mathcal{L}_{M^{n}} g^{n+1}-v \partial_{x} M^{n}\right] .
$$

Now, in the macroscopic part (23), the non-equilibrium flux $\partial_{x}\langle v m g\rangle$ is approximated by $\partial_{x}\left\langle v m g^{n+1}\right\rangle$. This allows us to obtain diffusion terms that are evaluated at $t_{n}$ instead of $t_{n-1}$. Then we obtain

$$
\frac{U^{n+1}-U^{n}}{\Delta t}+\partial_{x}\left\langle v m g^{n+1}\right\rangle=0 .
$$

Finally, we have the following proposition.

Proposition 4.1. The time discretization (24)-(25) gives at the limit $\varepsilon \rightarrow 0$ a numerical scheme which is consistent with diffusion equations (16).

Proof. Formally, $g^{n+1}$ can be determined from relation (24) as

$$
g^{n+1}=\left(I-\frac{\Delta t}{\varepsilon^{2}} \mathcal{L}_{M^{n}}\right)^{-1}\left[g^{n}-\frac{\Delta t}{\varepsilon^{2}} v \partial_{x} M^{n}-\frac{\Delta t}{\varepsilon}\left(I-\Pi_{M^{n}}\right)\left(v \partial_{x} g^{n}\right)+\frac{\Delta t}{\varepsilon} Q\left(g^{n}, g^{n}\right)\right] .
$$

Indeed, the fact that $\mathcal{L}_{M^{n}}$ is a non-positive self-adjoint operator on $L_{M^{n}}^{2}$ implies that $\left(I-\frac{\Delta t}{\varepsilon^{2}} \mathcal{L}_{M^{n}}\right)$ is a negative self-adjoint operator, hence invertible for every time step $\Delta t$, independtly of $\varepsilon$. By a simple expansion in $\varepsilon$, we get

$$
g^{n+1}=\mathcal{L}_{M^{n}}^{-1}\left(v \partial_{x} M^{n}\right)+O(\varepsilon) .
$$

Now, we proceed exactly as in the continuous case (see section 3.2): the previous expression is inserted in (25) to obtain the following time discrete numerical scheme:

$$
\frac{U^{n+1}-U^{n}}{\Delta t}+\partial_{x}\left\langle v m \mathcal{L}_{M^{n}}^{-1}\left(v \partial_{x} M^{n}\right)\right\rangle=O(\varepsilon) .
$$

This scheme is clearly consistent with (15) — which is nothing but system (16) — when $\varepsilon$ goes to 0 . 


\subsection{Space discretization}

In this section, we give a space discretization of the discrete scheme (24)-(25) such that it gives a consistent fully discrete approximation of the diffusion limit when $\varepsilon$ goes to 0 .

The idea consists in using two staggered spatial grids defined by $x_{i}=i \Delta x$ and $x_{i+\frac{1}{2}}=$ $\left(i+\frac{1}{2}\right) \Delta x$, where $\Delta x$ is a fixed spatial step. The non-equilibrium equation (24) is discretized at points $x_{i+\frac{1}{2}}$, while the macroscopic equation (25) is discretized at points $x_{i}$. Let us consider the approximations $U_{i}^{n} \approx U\left(t^{n}, x_{i}\right)$, and $g_{i+\frac{1}{2}}^{n}(v) \approx g\left(t^{n}, x_{i+\frac{1}{2}}, v\right)$. Now, to insure that the discretization of the non-equilibrium equation is stable in kinetic regimes (that is to say for $\varepsilon$ of the order of 1$)$, where convection is dominating, the term $\left(I-\Pi_{M}\right)\left(v \partial_{x} g^{n}\right)$ is discretized by a simple first order upwind scheme. On the other hand, the terms $v \partial_{x} M^{n}$ (in (24)) and $\partial_{x}\left\langle v m g^{n+1}\right\rangle$ (in (25)) give the diffusive terms at the limit $\varepsilon \rightarrow 0$ in (25): consequently, they are discretized by using central differences. Finally, we obtain the following scheme:

$$
\begin{aligned}
& \frac{g_{i+\frac{1}{2}}^{n+1}-g_{i+\frac{1}{2}}^{n}}{\Delta t}+\frac{1}{\varepsilon}\left(I-\Pi_{i+\frac{1}{2}}^{n}\right)\left[v^{+} \frac{g_{i+\frac{1}{2}}^{n}-g_{i-\frac{1}{2}}^{n}}{\Delta x}+v^{-} \frac{g_{i+\frac{3}{2}}^{n}-g_{i+\frac{1}{2}}^{n}}{\Delta x}\right]-\frac{1}{\varepsilon} Q\left(g_{i+\frac{1}{2}}^{n}, g_{i+\frac{1}{2}}^{n}\right) \\
& =\frac{1}{\varepsilon^{2}}\left[\mathcal{L}_{i+\frac{1}{2}}^{n} g_{i+\frac{1}{2}}^{n+1}-v \frac{M_{i+1}^{n}-M_{i}^{n}}{\Delta x}\right], \\
& \frac{U_{i}^{n+1}-U_{i}^{n}}{\Delta t}+\left\langle v m \frac{g_{i+\frac{1}{2}}^{n+1}-g_{i-\frac{1}{2}}^{n+1}}{\Delta x}\right\rangle=0 .
\end{aligned}
$$

where $v^{ \pm}=\frac{1}{2}(v \pm|v|)$. The linearized operator $\mathcal{L}_{i+\frac{1}{2}}^{n}$ and the projection $\Pi_{i+\frac{1}{2}}^{n}$ are defined by the averages $\mathcal{L}_{i+\frac{1}{2}}^{n}=\frac{\mathcal{L}\left(U_{i}^{n}\right)+\mathcal{L}\left(U_{i+1}^{n}\right)}{2}$ and $\Pi_{i+\frac{1}{2}}^{n}=\frac{\Pi_{M_{i}^{n}+\Pi_{M_{i+1}}}}{2}$.

The following proposition states that our scheme has the expected property.

Proposition 4.2. Let consider the following numerical scheme (27)-(28) for Kac equation (8). At the limit $\varepsilon \rightarrow 0$ we obtain the following numerical scheme:

$$
\frac{U_{i}^{n+1}-U_{i}^{n}}{\Delta t}+\frac{1}{\Delta x}\left\langle v m\left[\mathcal{L}_{i+\frac{1}{2}}^{-1}\left(v \frac{M_{i+1}^{n}-M_{i}^{n}}{\Delta x}\right)-\mathcal{L}_{i-\frac{1}{2}}^{-1}\left(v \frac{M_{i}^{n}-M_{i-1}^{n}}{\Delta x}\right)\right]\right\rangle=0
$$

which is a consistent approximation of the diffusion system (16). Moreover, the approximation of the diffusion term is second order in space.

Proof. The proof follows the same lines as in the continuous and the time semi-discrete cases (see the proof of proposition 4.1): from (27), we get

$$
g_{i+\frac{1}{2}}^{n+1}=\mathcal{L}_{i+\frac{1}{2}}^{-1}\left(v \frac{M_{i+1}^{n}-M_{i}^{n}}{\Delta x}\right)+O(\varepsilon)
$$

Using this expression in (28) and then passing to the limit directly gives (29). The second order accuracy can be easily checked by a classical Taylor expansion w.r.t $x$ for small $\Delta x$. 


\subsection{Velocity discretization}

In this section, we give a very simple velocity discretization of the time and space discrete scheme (27)-(28). The only difficulty is to correctly discretize the collision operator.

First, we propose a reduced formulation of this operator to reduce its complexity (the proof of the following result is given in appendix B).

Proposition 4.3. The Kac collision operator (2) can be written as

$$
\begin{aligned}
Q(f, f)(v)= & \frac{1}{\pi} \int_{0}^{+\infty} \int_{0}^{\frac{\pi}{4}}\left[\left(f\left(v^{\prime}\right)+f\left(-v^{\prime}\right)\right)\left(f\left(v_{*}^{\prime}\right)+f\left(-v_{*}^{\prime}\right)\right)-2 f(v)\left(f\left(v_{*}\right)+f\left(-v_{*}\right)\right)\right. \\
& \left.+\left(f\left(v^{\prime \prime}\right)+f\left(-v^{\prime \prime}\right)\right)\left(f\left(v_{*}^{\prime \prime}\right)+f\left(-v_{*}^{\prime \prime}\right)\right)-2 f(v)\left(f\left(v_{*}\right)+f\left(-v_{*}\right)\right)\right] d \theta d v_{*},
\end{aligned}
$$

where $\left(v^{\prime \prime}, v_{*}^{\prime \prime}\right)=\left(v \cos \theta+v_{*} \sin \theta, v \sin \theta-v_{*} \cos \theta\right)$ and $\left(v^{\prime}, v_{*}^{\prime}\right)$ are defined in (3).

Then we propose a discretization based on standard quadrature and interpolation formula. First, the velocity space is truncated to the interval $\left[v_{\min }, v_{\max }\right]$ where $v_{\min }=-v_{\max }$. Let us consider a discretization of this space velocity as $v_{p}=-v_{-p}=\left(p-\frac{1}{2}\right) \Delta v, p=1, \cdots, N$ where $\Delta v=\frac{v_{\max }}{N-\frac{1}{2}}$ is a fixed uniform step. We denote by $\theta_{k}=k \Delta \theta, k=0, \cdots, N t$ a discretization of the interval $\left[0, \frac{\pi}{4}\right.$ [ where $\Delta \theta=\frac{\pi}{4 N t}$ is a fixed step. For given discrete pre-collisional velocities $\left(v_{p}, v_{q}\right)$, the velocities $v_{p} \cos \theta_{k} \pm v_{q} \sin \theta_{k}$ and $v_{p} \sin \theta_{k} \pm v_{q} \cos \theta_{k}$ do not usually belong to the space velocity $\left[v_{\min }, v_{\max }\right]$. Thus, we introduce the sets of indexes of admissible angles associated to different couples of velocities $\left(v_{p}, v_{q}\right)$ as follows:

$$
\begin{aligned}
& \mathcal{C}_{p, q}^{\text {red }}=\left\{k=0, \cdots, N t: \max \left(\left|v_{p} \cos \theta_{k}-v_{q} \sin \theta_{k}\right|,\left|v_{p} \sin \theta_{k}+v_{q} \cos \theta_{k}\right|\right) \leq v_{\max }\right\} . \\
& \mathcal{D}_{p, q}^{r e d}=\left\{k=0, \cdots, N t: \max \left(\left|v_{p} \cos \theta_{k}+v_{q} \sin \theta_{k}\right|,\left|v_{p} \sin \theta_{k}-v_{q} \cos \theta_{k}\right|\right) \leq v_{\max }\right\}
\end{aligned}
$$

Now, in order to approximate $\left(v_{p} \cos \theta_{k}-v_{q} \sin \theta_{k}, v_{p} \sin \theta_{k}+v_{q} \cos \theta_{k}\right), k \in \mathcal{C}_{p, q}$ and $\left(v_{p} \cos \theta_{k}+\right.$ $\left.v_{q} \sin \theta_{k}, v_{p} \sin \theta_{k}-v_{q} \cos \theta_{k}\right), l \in \mathcal{D}_{p, q}$, our idea consists in replacing each velocity by the closest point in the velocity grid. More precisely, let introduce the even function $x \in \mathbb{R} \mapsto$ $\operatorname{Int}(x) \in \mathbb{Z}$ defined par the closest integer of $x$. Let $f_{q}$ and $Q_{p}$ be two approximations of $f\left(v_{q}\right)$ and $Q(f, f)\left(v_{q}\right),|q|=1, \cdots, N$ respectively. By writting

$$
\left(v_{p} \cos \theta_{k}-v_{q} \sin \theta_{k}, v_{p} \sin \theta_{k}+v_{q} \cos \theta_{k}\right)=\left(\left(p \cos \theta_{k}-q \sin \theta_{k}\right) \Delta v,\left(p \sin \theta_{k}+q \cos \theta_{k}\right) \Delta v\right),
$$

we obtain the following approximations

$$
\begin{aligned}
& f\left(v_{p} \cos \theta_{k}-v_{q} \sin \theta_{k}\right) \approx f_{\text {Int }\left(p \cos \theta_{k}-q \sin \theta_{k}\right)}, \\
& f\left(v_{p} \sin \theta_{k}+v_{q} \cos \theta_{k}\right) \approx f_{\text {Int }\left(p \sin \theta_{k}+q \cos \theta_{k}\right)} .
\end{aligned}
$$


In the same way, we get

$$
\begin{aligned}
f\left(v_{p} \cos \theta_{k}+v_{q} \sin \theta_{k}\right) & \approx f_{\text {Int }\left(p \cos \theta_{k}+q \sin \theta_{k}\right)}, \\
f\left(v_{p} \sin \theta_{k}-v_{q} \cos \theta_{k}\right) & \approx f_{\text {Int }\left(p \sin \theta_{k}-q \cos \theta_{k}\right)} .
\end{aligned}
$$

Finally, the complete discretization of the collision kac operator (in its reduced form (30)) is:

$$
Q_{p}(f, f)=\frac{1}{\pi} \sum_{q=1}^{N}\left[S_{p, q}^{1}+S_{p, q}^{2}\right] \Delta v
$$

with

$$
\begin{gathered}
S_{p, q}^{1}=\sum_{k \in \mathcal{C}_{p, q}^{r e d}}\left[\left(f_{\operatorname{Int}\left(p \cos \theta_{k}-q \sin \theta_{k}\right)}+f_{-\operatorname{Int}\left(p \cos \theta_{k}-q \sin \theta_{k}\right)}\right)\left(f_{\operatorname{Int}\left(p \sin \theta_{k}+q \cos \theta_{k}\right)}+f_{-\operatorname{Int}\left(p \sin \theta_{k}+q \cos \theta_{k}\right)}\right)\right. \\
-2 f(p)(f(q)+f(-q))] \Delta \theta,-N \leq p \leq N, \quad 1 \leq q \leq N, \\
S_{p, q}^{2}=\sum_{k \in \mathcal{D}_{p, q}^{r e d}}\left[\left(f_{\operatorname{Int}\left(p \cos \theta_{k}+q \sin \theta_{k}\right)}+f_{-\operatorname{Int}\left(p \cos \theta_{k}+q \sin \theta_{k}\right)}\right)\left(f_{\operatorname{Int}\left(p \sin \theta_{k}-q \cos \theta_{k}\right)}+f_{-\operatorname{Int}\left(p \sin \theta_{k}-q \cos \theta_{k}\right)}\right)\right. \\
-2 f(p)(f(q)+f(-q))] \Delta \theta,-N \leq p \leq N, \quad 1 \leq q \leq N,
\end{gathered}
$$

The velocity discretization of the linearized operator $\mathcal{L}_{M}$ is obtained simply by linearizing the approximation of the Kac operator given by (31)-(32). Then $\left(\mathcal{L}_{M^{n}} g\right)\left(v_{p}\right)$ is approximated by $\left(\mathcal{L}_{M^{n}} g\right)_{p}=2 Q_{p}\left(M^{n}, g\right)$, where $Q_{p}(f, g)$ is the polar form of the discrete collision operator $Q_{p}$ defined in(31)-(32).

Finally, our fully discrete scheme now reads:

$$
\begin{aligned}
& \frac{g_{i+\frac{1}{2}, p}^{n+1}-g_{i+\frac{1}{2}, p}^{n}}{\Delta t}+\frac{1}{\varepsilon}\left(\left(I-\Pi_{i+\frac{1}{2}}^{n}\right)\left[v^{+} \frac{g_{i+\frac{1}{2}}^{n}-g_{i-\frac{1}{2}}^{n}}{\Delta x}+v^{-} \frac{g_{i+\frac{3}{2}}^{n}-g_{i+\frac{1}{2}}^{n}}{\Delta x}\right]\right)_{p}-\frac{1}{\varepsilon} Q_{p}\left(g_{i+\frac{1}{2}}^{n}, g_{i+\frac{1}{2}}^{n}\right) \\
& =\frac{1}{\varepsilon^{2}}\left[\left(\mathcal{L}_{i+\frac{1}{2}}^{n} g_{i+\frac{1}{2}}^{n+1}\right)_{p}-v \frac{M_{i+1, p}^{n}-M_{i, p}^{n}}{\Delta x}\right] \\
& \frac{U_{i}^{n+1}-U_{i}^{n}}{\Delta t}+\left\langle v m \frac{g_{i+\frac{1}{2}}^{n+1}-g_{i-\frac{1}{2}}^{n+1}}{\Delta x}\right\rangle=0
\end{aligned}
$$

where $\langle\phi\rangle$ now stands for the quadrature formula $\sum_{p=-N}^{N} \phi_{p} \Delta v$. This quadrature allows to define $M_{i, p}^{n}$ as the discrete Maxwellian such that $\sum_{p=-N}^{N} m\left(v_{p}\right) M_{i}^{n} \Delta v=U_{i}^{n}$, as well as the projection $\Pi_{i+\frac{1}{2}}^{n}$ by using $(20)$.

At each time iteration and for each point $x_{i}$, the vector $\left(g_{i+\frac{1}{2}, p}^{n+1}\right)_{p=-N}^{N}$ is computed by solving a linear system with the matrix $I-\frac{\Delta t}{\varepsilon^{2}} \mathcal{L}_{i+\frac{1}{2}}^{n}$. While solving such a full system might be an important issue for realistic collision operators, we simply used the standard LU factorization in our numerical tests. 
Remark 4.1. Note that, in general, the velocity approximation (31)-(32) does not preserve the strong properties of the continuous collison operator: in particular, the conservation properties are only satisfied approximately up to $\Delta v$. Another consequence is that, it is not garanteed that the matrix $I-\frac{\Delta t}{\varepsilon^{2}} \mathcal{L}_{i+\frac{1}{2}}^{n}$ is symetric positive, even not invertible. However, in all our numerical tests, this matrix is never singular.

Remark 4.2. We have tried to construct a conservative approximation using the technique of Rogier and Schneider (see [24]) that was proposed for the Boltzmann equation. This approach consists, for each angle of rotation $\theta_{k}$, in using only the velocities $v_{k}, v_{l}$ such that the post-collisionnal velocities are nodes of the velocity grid. In other words, one have to select the intersection points of a cartesian grid with a circle. The formula we obtained is indeed conservative, but the number of velocities satisfying the previous property is very small in general. Consequently, the equilibrium states are defined by a very small number of constraints, and this approximation admits many parasit equilibrium states and then a wrong collision dynamics. This problem is due to the one-dimensional structure of the operator and is not observed for two- or three-dimensional operators like for Boltzmann. Another approach consists in using the Fourier transform (see [7]), which allows to obtain an expression that conserves the mass (but not the energy), that has a spectral accuracy, with a computational cost of $n \log n$ instead of $n^{2}$. However, since, in this work, our only goal is to illustrate our approach in a different context from that developped in [3], we did not tried to find an "optimal" velocity discretization: taking a large number of discrete velocities and angles make the scheme almost conservative. Of course, this might be really necessary with more complex and more realistic collisional operator, like the Boltzmann one, but we suggest below a simple "trick" that could fix the conservation problem when using the micro-macro decomposition.

For the micro-macro decomposition, the lack of conservation properties means that the mass and energy of $g_{i+\frac{1}{2}}^{n}$ are not exactly zero at every time step, and for every node $x_{i}$. However, our scheme can be slightly modified as follows: while in the continuous case $(I-$ $\Pi) Q=Q$ and $(I-\Pi) \mathcal{L}_{M}=\mathcal{L}_{M}$, this is not true in our discrete approximation. Then, let the projection $(I-\Pi)$ be applied to these collision operators to get the modified scheme (which is of course still a consistent approximation):

$$
\begin{aligned}
& \frac{g_{i+\frac{1}{2}, p}^{n+1}-g_{i+\frac{1}{2}, p}^{n}}{\Delta t}+\frac{1}{\varepsilon}\left(\left(I-\Pi_{i+\frac{1}{2}}^{n}\right)\left[v^{+} \frac{g_{i+\frac{1}{2}}^{n}-g_{i-\frac{1}{2}}^{n}}{\Delta x}+v^{-} \frac{g_{i+\frac{3}{2}}^{n}-g_{i+\frac{1}{2}}^{n}}{\Delta x}\right]\right)_{p} \\
& =\frac{1}{\varepsilon^{2}}\left[\left(\left(I-\Pi_{i+\frac{1}{2}}^{n}\right) \mathcal{L}_{i+\frac{1}{2}}^{n} g_{i+\frac{1}{2}}^{n+1}\right)_{p}-v \frac{M_{i+1, p}^{n}-M_{i, p}^{n}}{\Delta x}\right]+\frac{1}{\varepsilon}\left(\left(I-\Pi_{i+\frac{1}{2}}^{n}\right) Q\left(g_{i+\frac{1}{2}}^{n}, g_{i+\frac{1}{2}}^{n}\right)\right)_{p}, \\
& \frac{U_{i}^{n+1}-U_{i}^{n}}{\Delta t}+\left\langle v m \frac{g_{i+\frac{1}{2}}^{n+1}-g_{i-\frac{1}{2}}^{n+1}}{\Delta x}\right\rangle=0 .
\end{aligned}
$$

By construction, the moments of $g_{i+\frac{1}{2}}^{n}$ are always zero. 


\section{$5 \quad$ Numerical results}

In a first test, we validate the velocity discretization (31)-(32) of the collision operator. Then we present several numerical tests to confirm the properties of our micro-macro scheme (27)(28): it is uniformly accurate with respect to $\varepsilon$, and it preserves the diffusion limit.

For comparisons in the kinetic regime $\varepsilon=O(1)$, we compare our scheme to the following standard time explicit Euler method combined with a first order upwind spatial discretization for the transport terms, applied to the original Kac equation (8):

$$
\frac{f_{i, p}^{n+1}-f_{i, p}^{n}}{\Delta t}+\frac{1}{\varepsilon} \frac{v_{p}^{+}\left(f_{i, p}^{n}-f_{i-1, p}^{n}\right)+v_{p}^{-}\left(f_{i+1, p}^{n}-f_{i, p}^{n}\right)}{\Delta x}=\frac{1}{\varepsilon^{2}} Q_{p}\left(f_{i}^{n}, f_{i}^{n}\right) .
$$

This scheme cannot be AP in the diffusion regime, since it has the CFL constraint $\Delta t=$ $O\left(\varepsilon^{2}\right)$. Moreover, the upwind discretisation of the term $\frac{1}{\varepsilon} v \partial_{x} f$ introduces a numerical error of order $\frac{\Delta x}{\varepsilon}$, wich requires $\Delta x \ll \varepsilon$ when $\varepsilon$ is small. Consequently, in the diffusion regime, we compare our scheme to the following simple first order in time and second order in space approximation of the diffusion limit (16):

$$
\begin{aligned}
& \frac{1}{\Delta t}\left(\begin{array}{c}
\rho_{i}^{n+1}-\rho_{i}^{n} \\
E_{i}^{n+1}-E_{i}^{n}
\end{array}\right) \\
& -\frac{1}{\Delta x^{2}}\left(\begin{array}{c}
{\left[\frac{T_{i}^{n}+T_{i+1}^{n}}{2} \log \left(\frac{\rho_{i+1}^{n}}{\rho_{i}^{n}}\right)-\frac{T_{i-1}^{n}+T_{i}^{n}}{2} \log \left(\frac{\rho_{i}^{n}}{\rho_{i-1}^{n}}\right)\right]+T_{i+1}^{n}+T_{i-1}^{n}-2 T_{i}^{n}} \\
\frac{3}{2}\left\{\left[\frac{\left(T_{i}^{n}\right)^{2}+\left(T_{i+1}^{n}\right)^{2}}{2} \log \left(\frac{\rho_{i+1}^{n}}{\rho_{i}^{n}}\right)^{n}-\frac{\left(T_{i-1}^{n}\right)^{2}+\left(T_{i}^{n}\right)^{2}}{2} \log \left(\frac{\rho_{i}^{n}}{\rho_{i-1}^{n}}\right)\right]+\left(T_{i+1}^{n}\right)^{2}+\left(T_{i-1}^{n}\right)^{2}-2\left(T_{i}^{n}\right)^{2}\right\}
\end{array}\right) \\
& =0 \text {. }
\end{aligned}
$$

For clarity, we will refer to our asylmptotic preserving scheme (27)-(28) obtained by the micro-macro decomposition as $(A P)$, to the standard explicit scheme $(36)$ as $(E)$, to scheme (37) for the diffusion model as $(D)$.

\subsection{Homogeneous case}

Here, in order to test our velocity discretization, we consider the space homogeneous Kac collision operator that reads:

$$
\begin{aligned}
& \partial_{t} f=\frac{1}{\varepsilon^{2}} Q(f, f), \quad t \geq 0, \quad v \in\left[v_{\min }, v_{\max }\right] \\
& f_{\text {ini }}(v)=v^{2} \exp \left(-v^{2}\right)
\end{aligned}
$$

where $\left[v_{\min }, v_{\max }\right]=[-4.5,4.5]$ whose the discretization will be specified bellow. The exact solution of the classical problem (38)-(39) for which $v \in \mathbb{R}$ is obtained by the following 
analytical expression ( see Bobylev-Krook-Wu ([4],[18])):

$$
\begin{aligned}
& f(t, v)=\frac{1}{2}\left[\frac{3}{2}(1-C(t, \varepsilon) \sqrt{C(t, \varepsilon)})+(3 C(t, \varepsilon)-1) C(t, \varepsilon)^{\frac{3}{2}} v^{2}\right] \exp \left(-C(t, \varepsilon) v^{2}\right), \forall v \in \mathbb{R} \\
& C(t, \varepsilon)=\left[3-2 e^{-\frac{\sqrt{\pi}}{16} \frac{t}{\varepsilon^{2}}}\right]^{-1} .
\end{aligned}
$$

First we test our velocity discretization (31)-(32) with the standard explicite scheme $(E)$ which reads:

$$
f^{n+1}=f^{n}+\frac{\Delta t}{\varepsilon^{2}} Q\left(f^{n}, f^{n}\right) .
$$

In figure 1, we compare the numerical and exact solutions at time $t=0.2$ for different values of $\varepsilon: \varepsilon=1,0.3$ and 0.03 . The agreement is excellent, but the case $\varepsilon=0.03$ requires at least 240 velocities and 40 angles! With a coarser grid, the numerical solution can be not so accurate. The need for a very fine grid is a well known consequence of the nonconservative approximation we used: due to thhe CFL constraint on the time step, the number of iterations is larger for smaller $\varepsilon$, which amplifies the conervation errors.

Then we test the same velocity discretization with scheme (AP), which reads in the homogenous case:

$$
\begin{aligned}
& g^{n+1}=\left(I-\frac{\Delta t}{\varepsilon^{2}} \mathcal{L}_{M^{n}}\right)^{-1}\left[g^{n}+\frac{\Delta t}{\varepsilon} Q\left(g^{n}, g^{n}\right)\right] . \\
& U^{n+1}=U^{n} .
\end{aligned}
$$

In figure 2, we plot the distribution function (reconstructed as $f^{n}=M^{n}+\varepsilon g^{n}$ ), for the same time and values of $\epsilon$ as for the explicit scheme (E). Here, a very good agreement is observed for a slightly coarser grid: 100 velocities and 30 angles. This is due to two facts. First, the number of iterations to reach $t=0.2$ is independant of $\varepsilon$, so is the amplification of the errors. Second, in some sense, the conservation properties of $Q$ are already taken into account by the micro-macro formulation. In toher words, the moments $U$ are constant, and the conservation errors on $f$ are only due to the deviation part $g$, which is small for small $\varepsilon$.

\subsection{Sod problem}

In this section, we consider the classical Sod problem with the initial data for the density and temperature

$$
(\rho, T)=\left\{\begin{array}{l}
(1,0.1), \quad 0 \leq x \leq 1 \\
(0.25,0.02), \quad 1<x \leq 2 .
\end{array}\right.
$$

The distribution function is initialized with the Maxwellian states corresponding to this data. The space domain $[0,2]$ is discretized using 200 grid points, and the velocity domain $[-1.5,1.5]$ is discretized with 100 points. 
First, we analyze with the behavior of scheme $(A P)$ in different regimes $\left(\varepsilon_{n}=2^{-n}, n=\right.$ $1,2, \cdots)$. We plot at time $t=0.05$ in figure 3 the distribution function $f$ at $x=1$ as a function of the velocity $v \in[-1.5,1.5]$. Then, we observe that its profile converges towards a centered Maxwellian.

Now, for the same values of $\varepsilon$ we plot at time $t=0.05$, in figure 4$)-(5)$ the mass density, the energy, the temperature and the kinetic mean velocity obtained with scheme $(A P)$. Except for the mean velocity, in each figure the corresponding profile obtained by scheme $(D)$ for diffusion limit is also plotted. These figures shows that scheme $(A P)$ converges when $\varepsilon \rightarrow 0$ to the correct diffusion limit. Indeed, it gives in the diffusion regime $\varepsilon_{26}=1.49 \times 10^{-8}$ the same profiles of mass density, energy and temperature as the standard scheme $(D)$. Accordingly, we observe that the mean velocity $u=\langle v f\rangle$ tends to zero in the diffusion regime.

Finally, in order to show that the scheme $(A P)$ is also consistent in the kinetic regime with the Kac equation, we compare it to the explicit scheme $(E)$ for $\varepsilon=1$ at time $t=0.05$. In figure 6 , as expected, we can observe a very good agreement between the two schemes.

\section{Conclusion}

In this paper, we first used a micro-macro decomposition to transform the non-homogeneous Kac equation into an equivalent system coulping a kinetic part with a macroscopic one. Then a discretization of this system leads to a numerical scheme which is uniformly stable with respect to the Knudsen number. This scheme converges to a consistent discretization of the corresponding diffusion limit model when the Knudsen number tends to 0 and keeps a very good agreement with a standard scheme in the kinetic regime.

While our strategy has already been applied and validated on the BGK model in [3], the present work work shows that it is also efficient for the Kac collisional operator, which has a similar quadratic structure as the Boltzmann operator. This has been confirmed by formal analysis and numerical tests.

A next step will be the application of our approach to the real Boltzmann equation of rarfied gas dynamics. Conceptually, similar AP schemes can of course be written for the the Boltzmann equation, but the numerical implementation would require suitably accurate

velocity discretizations of the collision operator, as well as efficient linear solvers to compute the non-equilibrium part.

\section{References}

[1] C. Bardos, F. Golse, and D. Levermore. Fluid dynamic limits of kinetic equations. I. Formal derivations. J. Statist. Phys., 63(1-2):323-344, 1991. 
[2] M. Bennoune. Approximation numérique de quelques équations cinétiques préservant leurs asymptotiques fluides. PhD thesis, Université Paul Sabatier Toulouse 3, Toulouse, France, 2009. in french.

[3] Mounir Bennoune, Mohammed Lemou, and Luc Mieussens. Uniformly stable numerical schemes for the Boltzmann equation preserving the compressible Navier-Stokes asymptotics. J. Comput. Phys., 227(8):3781-3803, 2008.

[4] A. V. Bobylëv. Exact solutions of the Boltzmann equation. Dokl. Akad. Nauk SSSR, 225(6):1296-1299, 1975.

[5] R.-E. Caflisch, S. Jin, and G. Russo. Uniformly accurate schemes for hyperbolic systems with relaxation. SIAM J. Numer. Anal., 34(1):246-281, 1997.

[6] Laurent Desvillettes. About the regularizing properties of the non-cut-off Kac equation. Comm. Math. Phys., 168(2):417-440, 1995.

[7] Francis Filbet, Clément Mouhot, and Lorenzo Pareschi. Solving the Boltzmann equation in $N \log _{2} N$. SIAM J. Sci. Comput., 28(3):1029-1053 (electronic), 2006.

[8] E. Gabetta and L. Pareschi. About the non-cutoff Kac equation: uniqueness and asymptotic behaviour. Comm. Appl. Nonlinear Anal., 4(1):1-20, 1997.

[9] E. Gabetta, L. Pareschi, and G. Toscani. Relaxation schemes for nonlinear kinetic equations. SIAM J. Numer. Anal., 34(6):2168-2194, 1997.

[10] S. Jin. Efficient asymptotic-preserving (AP) schemes for some multiscale kinetic equations. SIAM J. Sci. Comput., 21(2):441-454 (electronic), 1999.

[11] S. Jin and C.-D Levermore. Numerical schemes for hyperbolic conservation laws with stiff relaxation terms. J. Comput. Phys., 126(2):449-467, 1996.

[12] S. Jin and L. Pareschi. Discretization of the multiscale semiconductor Boltzmann equation by diffusive relaxation schemes. J. Comput. Phys., 161(1):312-330, 2000.

[13] S. Jin and L. Pareschi. Asymptotic-preserving (AP) schemes for multiscale kinetic equations: a unified approach. In Hyperbolic problems: theory, numerics, applications, Vol. I, II (Magdeburg, 2000), volume 141 of Internat. Ser. Numer. Math., 140, pages 573-582. Birkhäuser, Basel, 2001.

[14] M. Kac. Probability and related topics in the physical sciences. Lectures in Applied Mathematics. Interscience Publishers London-New York, 1957.

[15] A. Klar. An asymptotic-induced scheme for nonstationary transport equations in the diffusive limit. SIAM J. Numer. Anal., 35(3):1073-1094 (electronic), 1998. 
[16] A. Klar. An asymptotic preserving numerical scheme for kinetic equations in the low Mach number limit. SIAM J. Numer. Anal., 36(5):1507-1527 (electronic), 1999.

[17] A. Klar. A numerical method for kinetic semiconductor equations in the drift-diffusion limit. SIAM J. Sci. Comput., 20(5):1696-1712 (electronic), 1999.

[18] M. Krook and T.T Wu. Formation of maxwellien tails. Phys. Rev. Lett., 36:1107-1109, 1976.

[19] M. Lemou and L. Mieussens. A new asymptotic preserving scheme based on micro-macro formulation for linear kinetic equations in the diffusion limit. 2008.

[20] H. P. McKean, Jr. Speed of approach to equilibrium for Kac's caricature of a Maxwellian gas. Arch. Rational Mech. Anal., 21:343-367, 1966.

[21] G. Naldi and L. Pareschi. Numerical schemes for kinetic equations in diffusive regimes. Appl. Math. Lett., 11(2):29-35, 1998.

[22] L. Pareschi and R.-E. Caflisch. An implicit Monte Carlo method for rarefied gas dynamics. I. The space homogeneous case. J. Comput. Phys., 154(1):90-116, 1999.

[23] L. Pareschi and G. Russo. Time relaxed Monte Carlo methods for the Boltzmann equation. SIAM J. Sci. Comput., 23(4):1253-1273 (electronic), 2001.

[24] François Rogier and Jacques Schneider. A direct method for solving the Boltzmann equation. Transport Theory Statist. Phys., 23(1-3):313-338, 1994.

\section{A Proof of proposition3.2}

Proof. The proof that $(U, g)$ is a solution of $(22)-(23)$ is nothing but the construction of system (22)-(23) detailed in section 3.1. For the converse statement, let $(U, g)$ be a solution of system (22)-(23) and consider the distribution function $f$ defined by $f=M+\varepsilon g$, where $M$ is the centered Maxwellian associated to $U$ by (6)-(7). We want to prove that $f$ satisfies (8). From (22), one can write

$$
\partial_{t} g+\frac{1}{\varepsilon^{2}} v \partial_{x} f=\frac{1}{\varepsilon^{2}} \mathcal{L}_{M} g+\frac{1}{\varepsilon} Q(g, g)+\frac{1}{\varepsilon} \Pi_{M}\left(v \partial_{x} g\right) .
$$

Then,

$$
\begin{aligned}
\partial_{t} f & +\frac{1}{\varepsilon} v \partial_{x} f=\frac{1}{\varepsilon} \mathcal{L}_{M} g+Q(g, g)+\frac{1}{\varepsilon} \Pi_{M}\left(v \partial_{x} f\right)+\partial_{t} M \\
& =\frac{1}{\varepsilon^{2}} Q(f, f)+\Pi_{M}\left(v \partial_{x} g\right)+\partial_{t} M
\end{aligned}
$$

On the other hand, we have $\Pi_{M}\left(v \partial_{x} g\right)+\partial_{t} M \in \mathcal{K} e r\left(\mathcal{L}_{M}\right)$ as a sum of elements of $\mathcal{K} e r\left(\mathcal{L}_{M}\right)$, and $\Pi_{M}\left(v \partial_{x} g\right)+\partial_{t} M \in \mathcal{R}\left(\mathcal{L}_{M}\right)$ thanks to $(23)$. Consequently, $\Pi_{M}\left(v \partial_{x} g\right)+\partial_{t} M=0$ and 
then $f$ satisfies (8). By construction, the initial condition is necessarily satisfied. Note also that the couple $(U, g)$ satisfies $U(t)=\langle m f(t)\rangle$ and $\langle m g(t)\rangle=0$ for every $t$.

\section{B Simplification of the collision integral}

Here, we give a proof of proposition 4.3. The integral with respect to $\left(v_{*}, \theta\right)$ in $[0,2 \pi[\times \mathbb{R}$ in (2) is simplified to an integral in $\left[0, \frac{\pi}{4}\left[\times \mathbb{R}^{+}\right.\right.$by using some symetries.

We introduce in all what follows the velocities $v^{\prime \prime}$ and $v_{*}^{\prime \prime}$ defined by

$$
\left(v^{\prime \prime}, v_{*}^{\prime \prime}\right)=\left(v \cos \theta+v_{*} \sin \theta, v \sin \theta-v_{*} \cos \theta\right)
$$

Let consider the decomposition of the following integral with respect to $\theta$ as:

$$
\int_{0}^{2 \pi} f\left(v^{\prime}\right) f\left(v_{*}^{\prime}\right) d \theta=\int_{0}^{\pi} f\left(v^{\prime}\right) f\left(v_{*}^{\prime}\right) d \theta+\int_{\pi}^{2 \pi} f\left(v^{\prime}\right) f\left(v_{*}^{\prime}\right) d \theta
$$

where $\left(v^{\prime}, v_{*}^{\prime}\right)$ is given by (3). By applying the variable change $\theta \leftrightarrow 2 \pi-\theta$ to the integral over $[\pi, 2 \pi[$ in the right hand side of $(46)$, we obtain

$$
\int_{0}^{2 \pi} f\left(v^{\prime}\right) f\left(v_{*}^{\prime}\right) d \theta=\int_{0}^{\pi}\left[f\left(v^{\prime}\right) f\left(v_{*}^{\prime}\right)+f\left(v^{\prime \prime}\right) f\left(-v_{*}^{\prime \prime}\right)\right] d \theta .
$$

In the same way, we get

$$
\begin{aligned}
\int_{0}^{2 \pi} f\left(v^{\prime}\right) f\left(v_{*}^{\prime}\right) d \theta & =\int_{0}^{\frac{\pi}{2}}\left[f\left(v^{\prime}\right) f\left(v_{*}^{\prime}\right)+f\left(v^{\prime \prime}\right) f\left(-v_{*}^{\prime \prime}\right)\right] d \theta+\int_{\frac{\pi}{2}}^{\pi}\left[f\left(v^{\prime}\right) f\left(v_{*}^{\prime}\right)+f\left(v^{\prime \prime}\right) f\left(-v_{*}^{\prime \prime}\right)\right] d \theta . \\
& =\int_{0}^{\frac{\pi}{2}}\left[f\left(v^{\prime}\right) f\left(v_{*}^{\prime}\right)+f\left(v^{\prime \prime}\right) f\left(-v_{*}^{\prime \prime}\right)+f\left(-v^{\prime \prime}\right) f\left(v_{*}^{\prime \prime}\right)+f\left(-v^{\prime}\right) f\left(-v_{*}^{\prime}\right)\right] d \theta,(48)
\end{aligned}
$$

thanks to the variable change $\theta \leftrightarrow \pi-\theta$. Now, decomposing integral (48) and using the variable change $\theta \leftrightarrow \frac{\pi}{2}-\theta$, we the obtain

$$
\begin{aligned}
\int_{0}^{2 \pi} f\left(v^{\prime}\right) f\left(v_{*}^{\prime}\right) d \theta= & \int_{0}^{\frac{\pi}{4}}\left[f\left(v^{\prime}\right) f\left(v_{*}^{\prime}\right)+f\left(v^{\prime \prime}\right) f\left(-v_{*}^{\prime \prime}\right)+f\left(-v^{\prime \prime}\right) f\left(v_{*}^{\prime \prime}\right)+f\left(-v^{\prime}\right) f\left(-v_{*}^{\prime}\right)\right] d \theta \\
& +\int_{\frac{\pi}{4}}^{\frac{\pi}{2}}\left[f\left(v^{\prime}\right) f\left(v_{*}^{\prime}\right)+f\left(v^{\prime \prime}\right) f\left(-v_{*}^{\prime \prime}\right)+f\left(-v^{\prime \prime}\right) f\left(v_{*}^{\prime \prime}\right)+f\left(-v^{\prime}\right) f\left(-v_{*}^{\prime}\right)\right] d \theta . \\
= & \int_{0}^{\frac{\pi}{4}}\left[f\left(v^{\prime}\right) f\left(v_{*}^{\prime}\right)+f\left(v^{\prime \prime}\right) f\left(-v_{*}^{\prime \prime}\right)+f\left(-v^{\prime \prime}\right) f\left(v_{*}^{\prime \prime}\right)+f\left(-v^{\prime}\right) f\left(-v_{*}^{\prime}\right)\right. \\
& \left.+f\left(v_{*}^{\prime \prime}\right) f\left(v^{\prime \prime}\right)+f\left(v_{*}^{\prime}\right) f\left(-v^{\prime}\right)+f\left(-v_{*}^{\prime}\right) f\left(v^{\prime}\right)+f\left(-v_{*}^{\prime \prime}\right) f\left(-v^{\prime \prime}\right)\right] d \theta .(49)
\end{aligned}
$$

Finally, we get the folowing expression

$$
\int_{0}^{2 \pi} f\left(v^{\prime}\right) f\left(v_{*}^{\prime}\right) d \theta=\int_{0}^{\frac{\pi}{4}} g\left(v, v_{*}, \theta\right) d \theta
$$


where the function $g\left(v, v_{*}, \theta\right)$ is given by

$$
g\left(v, v_{*}, \theta\right)=\left(f\left(v^{\prime}\right)+f\left(-v^{\prime}\right)\right)\left(f\left(v_{*}^{\prime}\right)+f\left(-v_{*}^{\prime}\right)\right)+\left(f\left(v^{\prime \prime}\right) f\left(-v^{\prime \prime}\right)\right)\left(f\left(v_{*}^{\prime \prime}\right)+f\left(-v_{*}^{\prime \prime}\right)\right)
$$

By reporting expression (50)-(51) in (2), we have

$$
Q(f, f)=\frac{1}{2 \pi} \int_{0}^{\frac{\pi}{4}} \int_{\mathbb{R}} g\left(v, v_{*}, \theta\right) d \theta d v_{*}-\frac{1}{2 \pi} \int_{0}^{2 \pi} \int_{\mathbb{R}} f(v) f\left(v_{*}\right) d \theta d v_{*} .
$$

We note that the function $v_{*} \mapsto g\left(v, v_{*}, \theta\right)$ is even, and thanks to the variable change $v_{*} \leftrightarrow$ $-v_{*}$, we obtain the final new formulation of the Kac operator given by

$$
\begin{aligned}
Q(f, f)(v)= & \frac{1}{\pi} \int_{0}^{+\infty} \int_{0}^{\frac{\pi}{4}}\left[\left(f\left(v^{\prime}\right)+f\left(-v^{\prime}\right)\right)\left(f\left(v_{*}^{\prime}\right)+f\left(-v_{*}^{\prime}\right)\right)-2 f(v)\left(f\left(v_{*}\right)+f\left(-v_{*}\right)\right)\right. \\
& \left.+\left(f\left(v^{\prime \prime}\right)+f\left(-v^{\prime \prime}\right)\right)\left(f\left(v_{*}^{\prime \prime}\right)+f\left(-v_{*}^{\prime \prime}\right)\right)-2 f(v)\left(f\left(v_{*}\right)+f\left(-v_{*}\right)\right)\right] d \theta d v_{*} .
\end{aligned}
$$




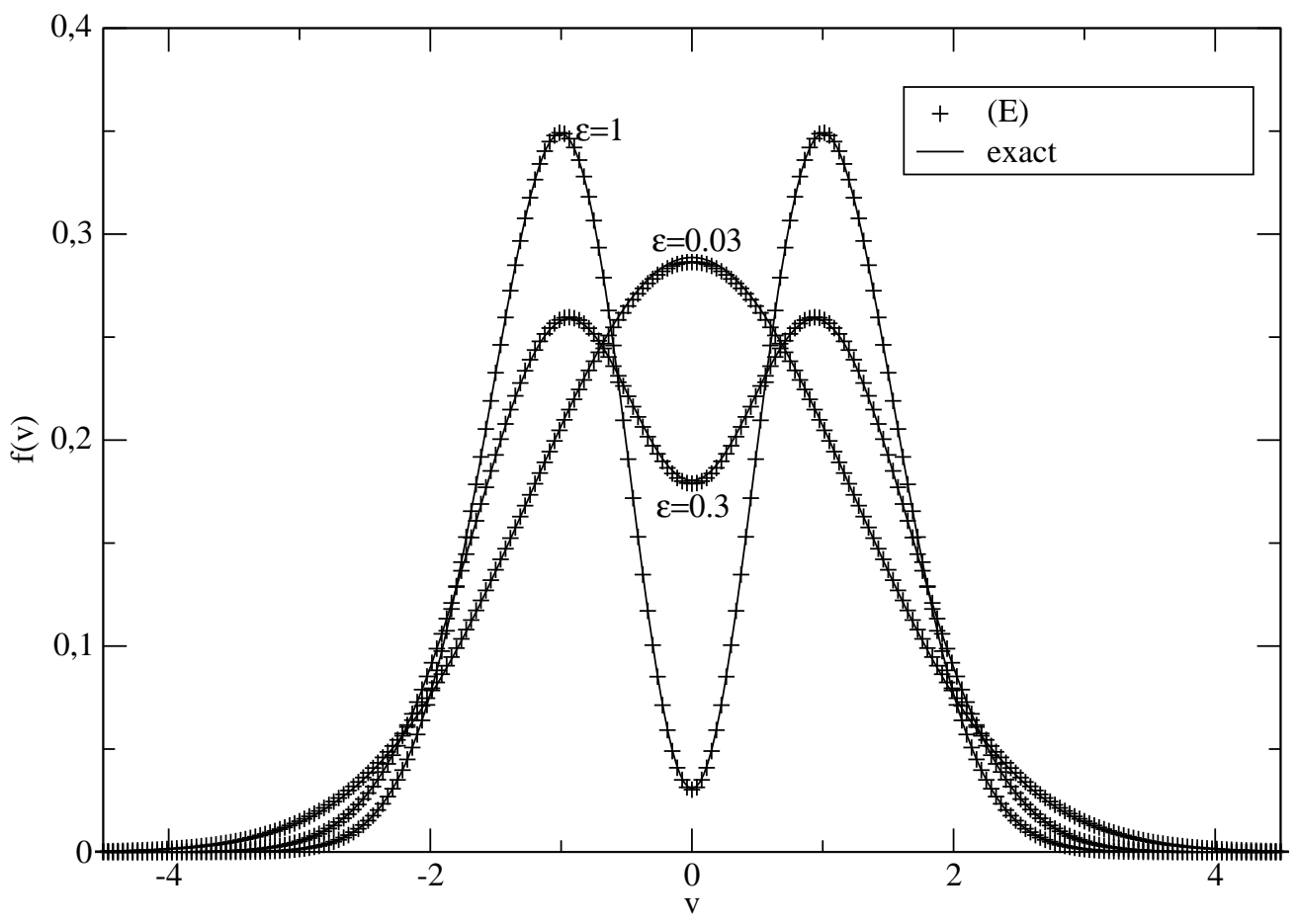

Figure 1: Homogeneous Kac equation: distribution function at time $t=0.2$ as a function of the velocity $v \in[-4.5,4.5]$ for $\varepsilon=1,0.3,0.03$ given by the exact solution and by the explicit scheme $(E)$. Velocity approximation with 240 discrete velocities and 40 discrete angles.

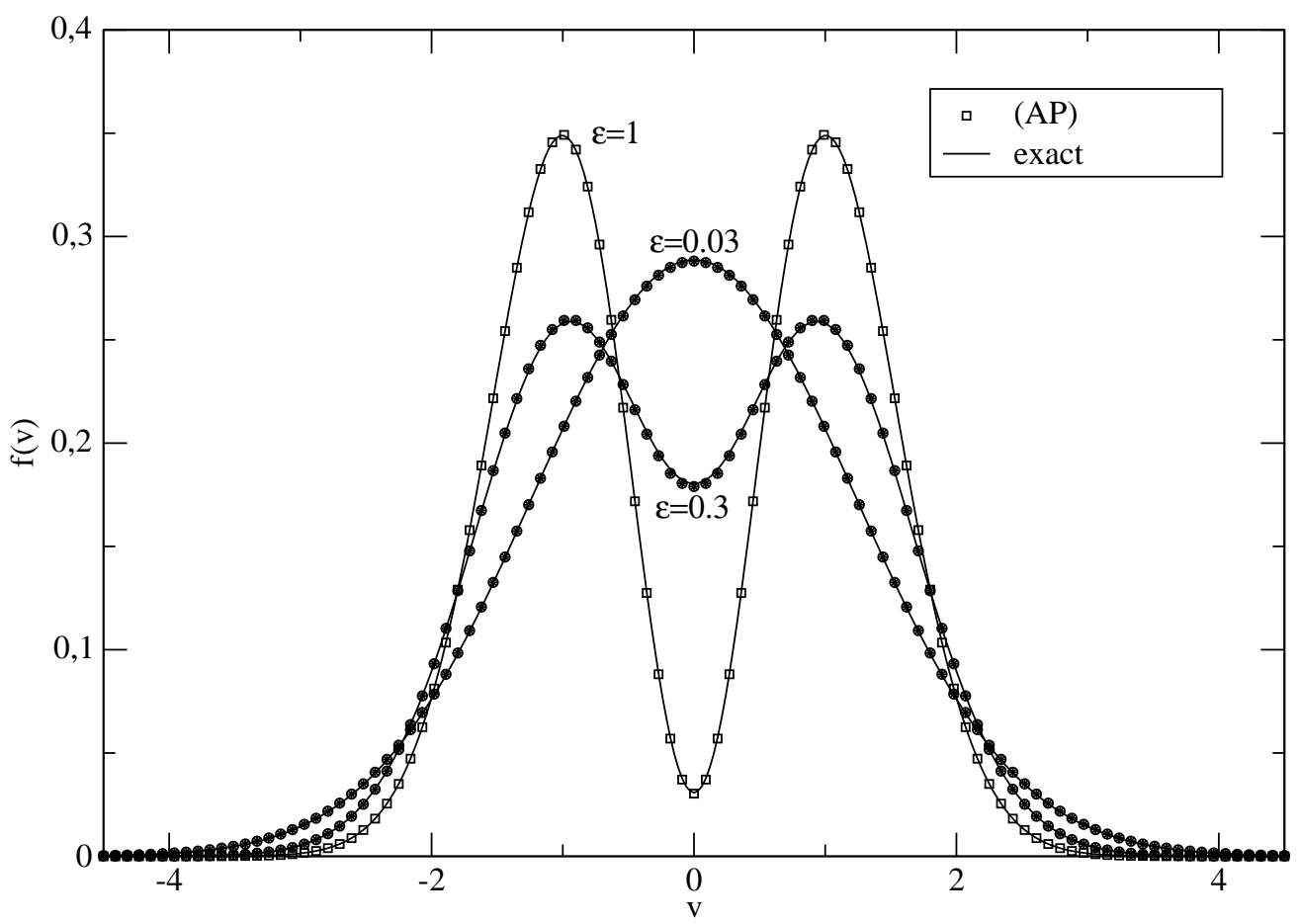

Figure 2: Homogeneous Kac equation: distribution function at time $t=0.2$ as a function of the velocity $v \in[-4.5,4.5]$ for $\varepsilon=1,0.3,0.03$ given by the exact solution and by scheme $(A P)$. Velocity approximation with 100 discrete velocities and 30 discrete angles. 


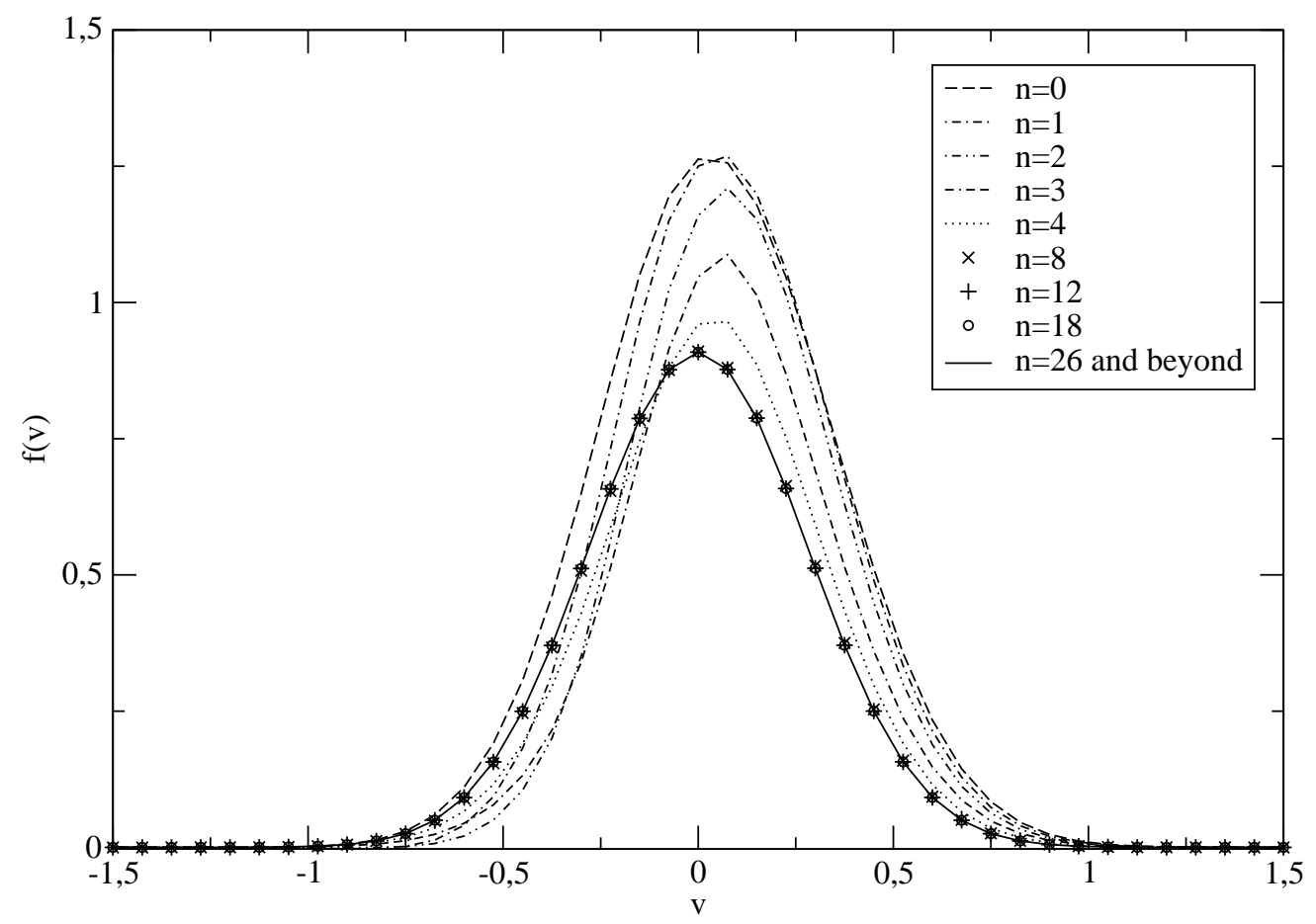

Figure 3: Sod problem: distribution function $f$ at time $t=0.05$ and $x=1$ as a function of the velocity $v \in[-1.5,1.5]$ given by scheme $(A P)$, for different values of $\varepsilon_{n}=2^{-n}$ : for the rarefied regime $\left(\varepsilon_{0}=1, \varepsilon_{1}=0.5, \varepsilon_{2}=0.25\right)$, the intermediate regime $\left(\varepsilon_{3}=0.125\right.$, $\left.\varepsilon_{4}=6.25 \times 10^{-2}\right)$ and diffusion regime $\left(\varepsilon_{8}=3.9 \times 10^{-3}, \varepsilon_{12}=2.44 \times 10^{-4}, \varepsilon_{18}=3.81 \times 10^{-6}\right.$, and $\left.\varepsilon \leq \varepsilon_{26}=1.49 \times 10^{-8}\right)$ in which the function $f$ becomes a centered Maxwellian. 

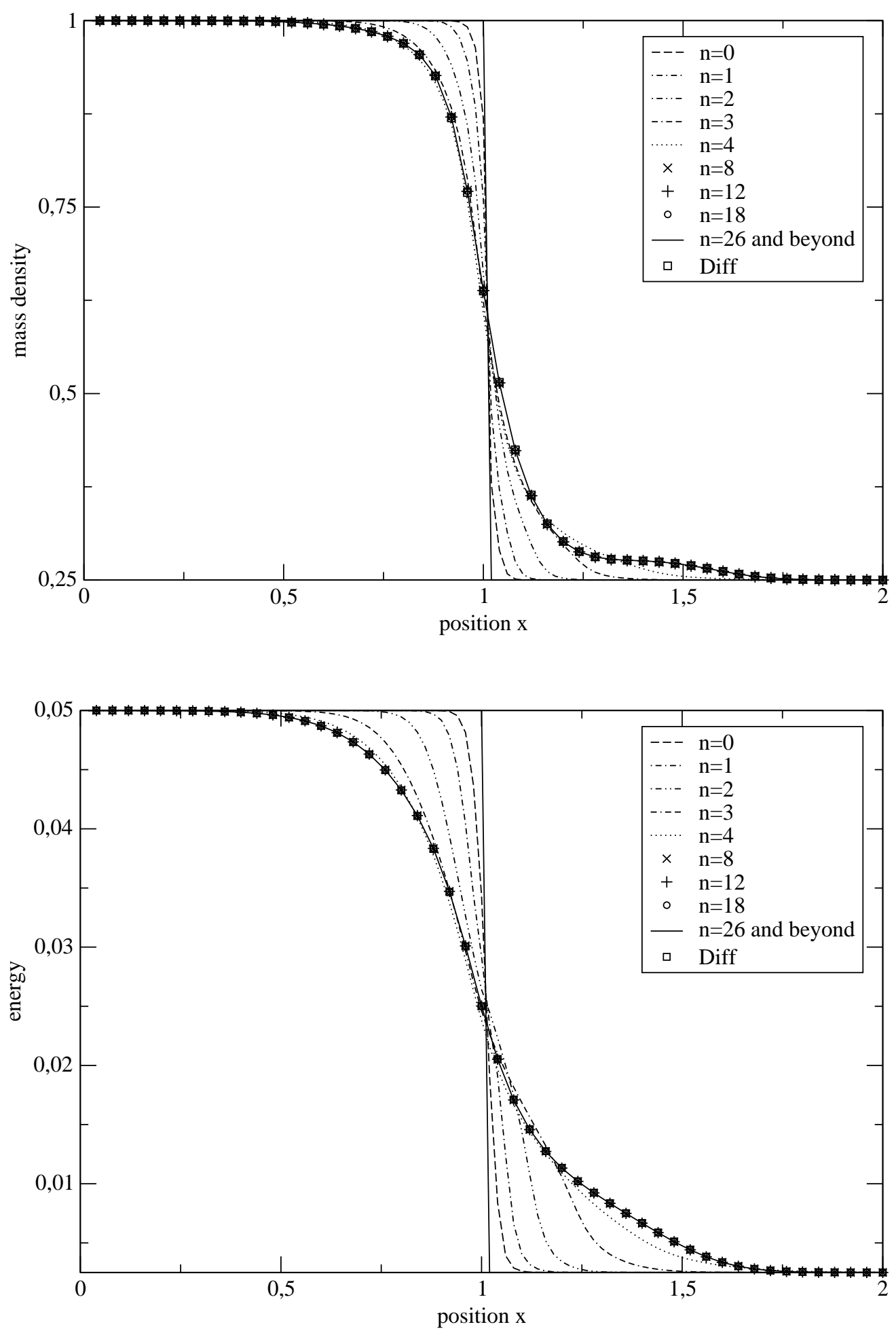

Figure 4: Sod problem: mass (top) and energy (bottom) densities as functions of $x \in[0,2]$ at time $t=0.05$, given by scheme $(A P)$. Profiles of $\rho$ for different values of $\varepsilon_{n}=2^{-n}$ : for the rarefied regime $\left(\varepsilon_{0}=1, \varepsilon_{1}=0.5, \varepsilon_{2}=0.25\right)$, the intermediate regime $\left(\varepsilon_{3}=0.125\right.$, $\left.\varepsilon_{4}=6.25 \times 10^{-2}\right)$ and diffusion regime $\left(\varepsilon_{8}=3.9 \times 10^{-3}, \varepsilon_{12}=2.44 \times 10^{-4}, \varepsilon_{18}=3.81 \times 10^{-6}\right.$, and for $\varepsilon \leq \varepsilon_{26}=1.49 \times 10^{-8}$ ) for which the2profile are the same as the ones obtained by scheme $(D)$ for the diffusion equation. 

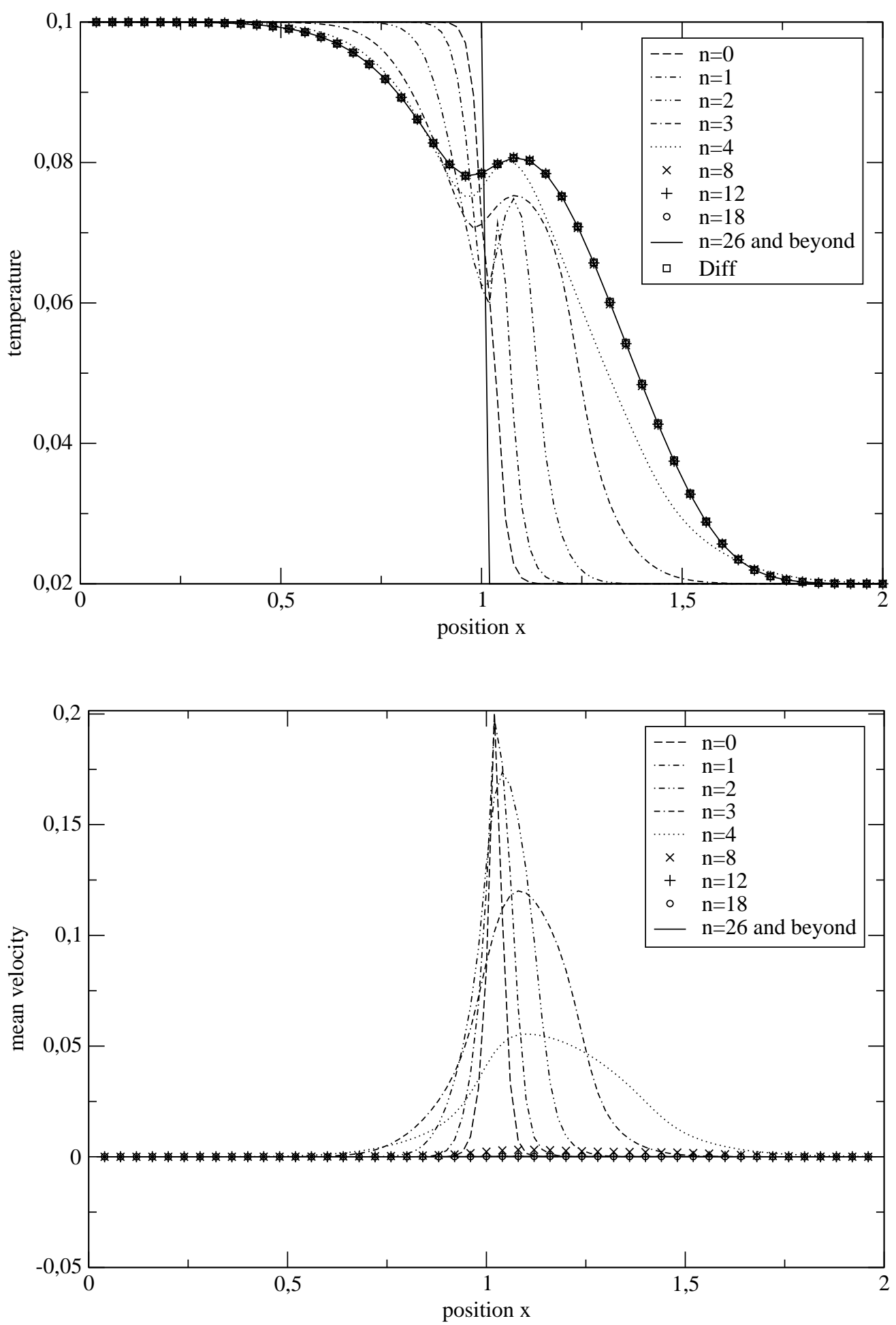

Figure 5: Sod problem: temperature (top) and velocity (bottom) as functions of $x \in[0,2]$ at time $t=0.05$, given by the scheme $(A P))$. Profils of $T$ for different values of $\varepsilon_{n}=2^{-n}$ : for the rarefied regime $\left(\varepsilon_{0}=1, \varepsilon_{1}=0.5, \varepsilon_{2}=0.25\right)$, the intermediate regime $\left(\varepsilon_{3}=0.125\right.$, $\left.\varepsilon_{4}=6.25 \times 10^{-2}\right)$ and diffusion regime $\left(\varepsilon_{8}=3.9 \times 10^{-3}, \varepsilon_{12}=2.44 \times 10^{-4}, \varepsilon_{18}=3.81 \times 10^{-6}\right.$, and for $\varepsilon \leq \varepsilon_{26}=1.49 \times 10^{-8}$ ) for which the 2 grofiles are the same as the ones obtained by scheme $(D)$ for the diffusion equation. 

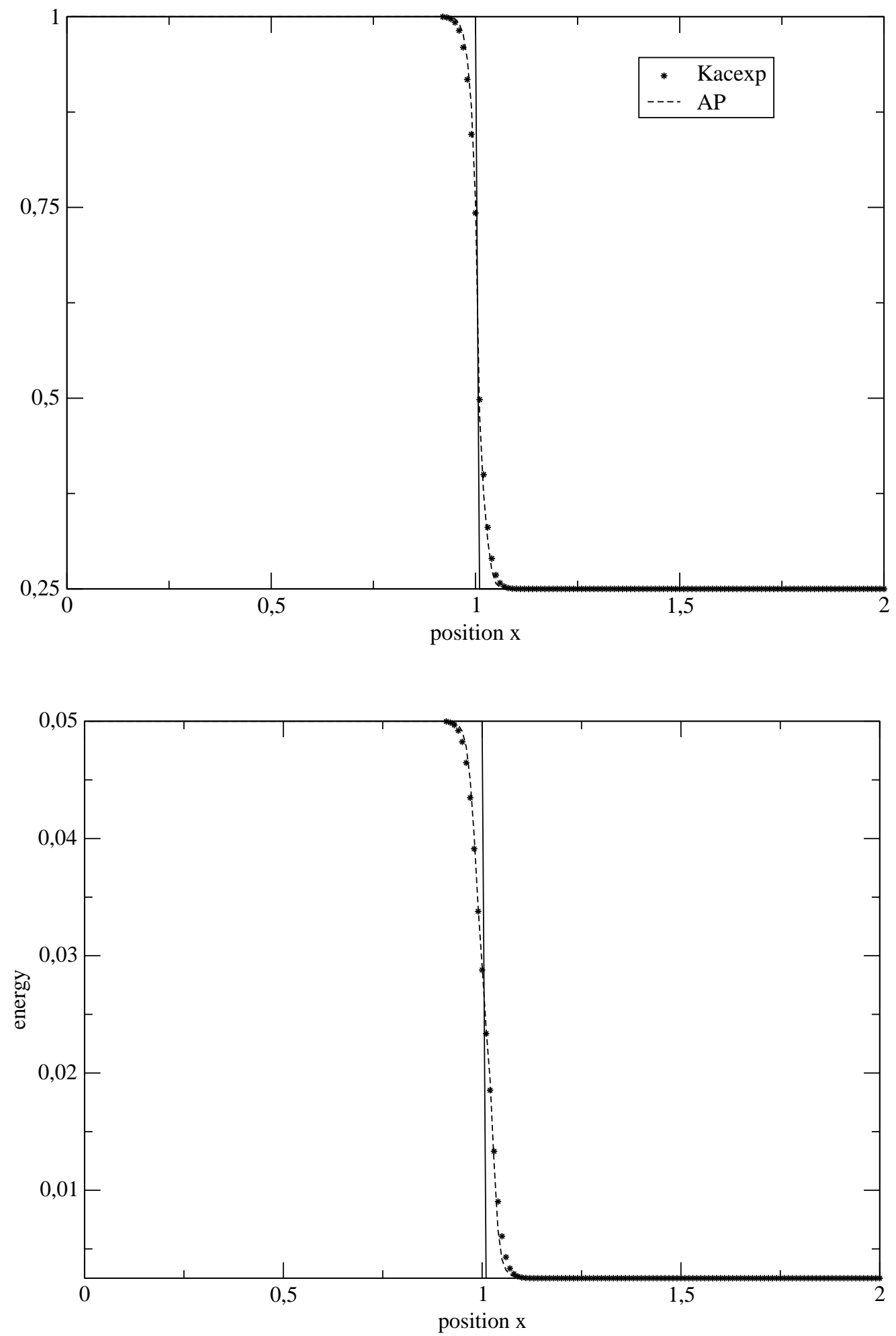

Figure 6: Sod problem: mass (top) and energy (bottom) densities at time $t=0.05$ as functions of the position $x \in[0,2]$ obtained by scheme $(A P)$ and $(E)$ in kinetic regime $\varepsilon=1$. 